\title{
Imagem corporal em mulheres adultas vs. meia-idade e idosas praticantes e não praticantes de hidroginástica
}

\author{
Body image in adult vs. middle-aged and elderly women practitioners and \\ non-practitioners of hydro gymnastics
}

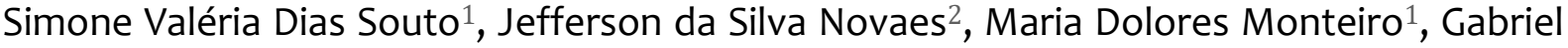
Rodrigues Neto ${ }^{2,3 *}$, Maria Isabel Mourão Carvalhal ${ }^{1}$, Eduarda Coelho ${ }^{1}$

ARTIGO ORIGINAL | ORIGINAL ARTICLE

\begin{abstract}
O objetivo do presente estudo foi comparar a imagem corporal de mulheres adultas $v s$. meia-idade e idosas praticantes e não praticantes de hidroginástica. A amostra foi constituída por 300 mulheres brasileiras com idades compreendidas entre 20 e 83 anos (48.96 \pm 15.41$)$, divididas em quatro grupos: Grupo $1=75$ mulheres adultas não praticantes com idades entre 20 e 49 anos; Grupo $2=75$ mulheres de meia-idade e idosas não praticantes com idades entre 50 e 82 anos; Grupo $3=75$ mulheres de meia-idade e idosas praticantes com idade entre 50 e 83 anos e Grupo $4=75$ mulheres adultas praticantes com idade entre 20 e 49 anos. O instrumento utilizado para determinar à imagem corporal actual e desejada foi a Escala de Desenhos de Silhuetas descrita por Stunkard. Os resultados demonstram que não houve diferenças significativas com a insatisfação da imagem corporal entre os grupos de mulheres adultas vs. meia-idade e idosas $v s$. praticantes $v s$. não praticantes $(p>0.05)$. Entretanto, houve diferenças significativas entre a insatisfação com a imagem corporal actual vs. ideal para todos os grupos do estudo $(p<0.05)$. Conclui-se que a idade e a prática da hidroginástica não são factores determinantes na percepção que as mulheres têm da imagem corporal.

Palavras-chave: imagem corporal, actividade física, hidroginástica, mulheres.
\end{abstract}

ABSTRACT

The aim of this study was to compare the body image of adult vs. middle-aged and elderly women practitioners and non-practitioners of hydro gymnastics. The sample consisted of 300 Brazilian women aged between 20 and 83 years $(48.96 \pm 15.41)$, divided into four groups: Group $1=75$ adult women not practitioners aged between 20 and 49 years; Group $2=75$ middle-aged and elderly women not practitioners aged 50 to 82 years; Group $3=75$ middle-aged and elderly women practitioners aged between 50 and 83 years and Group $4=75$ practitioners adult women aged between 20 and 49 years. The instrument used to determine the current and desired body image was the Scale Drawings of silhouettes described by Stunkard. The results showed no significant differences with body image dissatisfaction among groups of adults vs. middle-aged and elderly vs. practitioners vs. non-practicing ( $>0.05)$. However, there were significant differences between the dissatisfaction with the actual body image vs. ideal for all study groups $(\mathrm{p}<0.05)$. We conclude that the age and the practice of hydro gymnastics are not determining factors in the perception that women have body image.

Keywords: body image, physical activity, hydro gymnastics, women.

Artigo recebido a 06.09.2014; Aceite a 27.08.2015

${ }^{1}$ Departamento de Ciências do Desporto Exercício e Saúde, Universidade de Trás-os-Montes e Alto Douro, UTAD, Vila Real, Portugal.

${ }^{2}$ Departamento de Ginástica, Universidade Federal do Rio de Janeiro, UFRJ, Rio de Janeiro, Brasil

${ }^{3}$ Departmento de Educação Física, Programa Associado de Pós-Graduação em Educação Física UPE/UFPB, Universidade Federal da Paraíba, UFPB, João Pessoa, Brasil

* Autor correspondente: Departamento de Educação Física - Programa Associado de Pós-Graduação em Educação Física UPE/UFPB, Universidade Federal da Paraíba, João Pessoa, Paraíba, Brasil. E-mail: gabrielrodrigues_1988@hotmail.com 


\section{INTRODUÇÃO}

A imagem corporal (IC) é uma construção multidimensional que descreve amplamente as representações internas da estrutura corporal e da percepção física, em relação a nós mesmos e aos outros (Cash \& Pruzinsky, 1990). Assim, a interação das vivencias motoras e as dimensões socioculturais estabelecem parâmetros nos quais permite o indivíduo o constructo da imagem corporal (IC) (Cash \& Pruzinsky, 1990; Scatolin, 2012). Em idades mais avançadas são evidentes as alterações morfológicas e a insatisfação com a IC (Hausenblas \& Fallon, 2006; Matsuo, Velardi, Brandão, \& Jesus, 2007; Sato, Kaneda, Wakabayashi, \& Nomura, 2007), pois normalmente, os idosos associam a velhice à idéia de deterioração física e mental (Volkwein \& McConatha, 1997). Nesse contexto, uma das mais evidentes alterações que acontecem com o aumento da idade é as mudança nas dimensões corporais. A estatura sofre diminuição em função da compressão vertebral, do estreitamento dos discos e da cifose. Ocorre também à diminuição da massa magra, aumento no percentual de gordura e diminuição da densidade óssea (Kura, Ribeiro, Niquetti, \& Tourinho Filho, 2006), tais alterações podem influenciar negativamente na auto-avaliação da IC.

Estas distorções podem melhorar à medida que o individuo vai envelhecendo por meio da prática sistemática dos exercícios físicos (Hausenblas \& Fallon, 2006; Ricciardelli, McCabe, \& Banfield, 2000). Nesse sentido, a prática do exercício físico pode melhorar a qualidade de vida dos praticantes em virtude dos acréscimos da força muscular, da resistência muscular localizada, da flexibilidade, da capacidade cardiorrespiratória e a redução da composição corporal (ACSM, 2011). Estas melhorias podem promover alterações positivas da percepção da IC tanto em mulheres jovens como em mulheres adultas, de meia idade e idosas. Vale destacar que na mulher a IC está relacionada com factores multifacetados que estão na razão directa com conceitos socioculturais (Veggi, Lopes, Faerstein, \& Sichieri, 2004), ou seja, o processo de formação da IC pode ser influenciado pela mídia, família, amigos, meios de comunicação, idade e pelo sexo, bem como pela relação do corpo com os processos cognitivos como crença, valores e atitudes inseridos em uma cultura (Ricciardelli et al. 2000; Volkwein \& McConatha, 1997), no qual as mulheres estão mais susceptíveis a essas cobranças e, portanto, apresentam maiores graus de insatisfaçãocom a IC.

Baum (2000) e Etchepare, Pereira, Graup e Zinn (2003) esclarecem que a hidroginástica pode ser uma atividade de características agradáveis que pode influenciar na percepção da IC devido às alterações na massa corporal, favorecendo também o convívio social. A hidroginástica é um exercício físico muito procurado pelos adultos mais velhos $\mathrm{e}$ apresenta benefícios como a redução da frequência cardíaca, o aumento do $\mathrm{VO}_{2}$ máximo, da massa muscular, da amplitude articular, do bem-estar físico e psíquico e a função cognitiva (Assis, Junior, Santos, \& Navarro, 2007; Sato, Seko, Hashitomi, Sengoku, \& Nomura, 2015; Schuch et al., 2014). Todavia, ao rever a literatura pertinente observou-se que apenas um estudo avaliou a prática da hidroginástica sobre a IC (Mazo, Cardoso, \& Aguiar, 2006), este estudo verificou a auto-estima e a auto-imagem e a sua relação com os fatores motivacionais de ingresso e de permanência dos idosos em um programa de hidroginástica. Os autores, concluiram que o programa de hidroginástica melhora a autoimagem e auto-estima dos idosos, além de ser um fator que os mantém no programa. Entretanto, nenhum estudo comparou a IC em mulheres praticantes e não praticantes de hidroginastica em diferentes faixas etarias.

Diante do exposto, o presente estudo tem como objetivo comparar a imagem corporal de mulheres adultas $v s$. meia-idade e idosas praticantes e não praticantes de hidroginástica.

\section{MÉTODO}

\section{Amostra}

$\mathrm{O}$ presente estudo caracteriza-se como sendo de corte transversal. Participaram do estudo 300 mulheres com idades entre 20 e 83 
anos, saudáveis e residentes no Município de Montes Claros - Minas Gerais, Brasil. As mulheres, praticantes de hidroginástica, foram seleccionadas aleatoriamente em academias e as mulheres que não praticavam hidroginástica foram seleccionados nos postos de saúde e nos centros de convívio do Município. Após a selecção, as mulheres voluntárias foram distribuídas por grupos etários e pela prática ou não de hidroginástica, conforme segue: Grupo $1=75$ mulheres adultas não praticantes com idades entre 20 e 49 anos; Grupo $2=75$ mulheres de meia-idade e idosas não praticantes com idades entre 50 e 82 anos; Grupo $3=75$ mulheres de meia-idade e idosas praticantes com idade entre 50 e 83 anos e Grupo $4=75$ mulheres adultas praticantes com idade entre 20 e 49 anos.

Todas as mulheres foram informadas sobre o objetivo do estudo, tendo assinado um termo de consentimento livre esclarecido em conformidades com os princípios éticos presentes na Declaração de Helsínquia e na Resolução no 466/12 do Conselho Nacional de Saúde.

\section{Instrumentos}

\section{Medidas Antropométricas}

A estatura e a massa corporal foram medidas com precisão de $0.5 \mathrm{~cm} \quad$ e $0.1 \mathrm{~kg}$, respectivamente, utilizando um estadiômetro e uma balança da marca SECA ${ }^{\circledR}$. O índice de massa corporal (IMC) foi calculado em kg.m ${ }^{-2}$.

\section{Escala de Silhuetas - imagem corporal}

Para a identificação da imagem corporal foi utilizada a escala de Silhuetas proposta por Stunkard, Sørensen, e Schulsinger (1983). Esta escala é um conjunto de nove imagens do corpo humano, numeradas de 1 a 9 e compostas por duas perguntas: Qual é a silhueta que melhor representa a sua aparência física atualmente? Qual é a silhueta que você gostaria de ter? Para verificar a insatisfação corporal, utilizou-se a diferença entre a silhueta atual (SA) e silhueta ideal (SI), apontadas pelo indivíduo. O avaliador isentou-se de opinião na escolha das silhuetas. A avaliação da silhueta foi realizada antes das medidas a massa corporal e estatura, e foram tomadas no mesmo dia e horário pelo mesmo avaliador.

\section{Análise Estatística}

Utilizou-se o programa estatístico SPSS for windows versão 14.0 para analise de todos os dados. Os resultados foram apresentados pela média e desvio padrão. Para a comparação da massa corporal, estatura e IMC entre os grupos foi utilizada a anova One-way, com aplicação do post hoc de Tukey. Para comparação da imagem corporal (SA - SI) foi utilizado o teste wilcoxon para amostras pareadas e aplicação do teste jonckheere-terpstra, para analisar possíveis diferenças entre os grupos, com subsequente análise das diferenças entre a SA e a SI. Para a análise da relação entre a imagem corporal das mulheres (adultas vs. Meia-idade e idosas $v \boldsymbol{s}$. Praticantes vs. Não praticantes) foi utilizado o coeficiente de associação de Kappa. O nível de significância adotada foi de $p<0.05$.

\section{RESULTADOS}

$\mathrm{Na}$ comparação das variáveis antropométricas entre os grupos, observou-se diferença significativa $(p<0.05)$ para massa corporal de mulheres não praticantes (Grupo 1 $=67.65 \pm 10.32 \mathrm{~kg}$; Grupo $2=68.15 \pm 11.23$ $\mathrm{kg}$ ) vs. praticantes (Grupo $3=63.10 \pm 10.13$ $\mathrm{kg}$; Grupo $4=63.86 \pm 11.57 \mathrm{~kg}$ ). Bem como, existiu diferenças significativas entre o Grupo 4 $\left(24.50 \pm 4.26 \mathrm{~kg} / \mathrm{m}^{2}\right)$ vs. Grupo $1(26.66 \pm$ $\left.4.58 \mathrm{~kg} / \mathrm{m}^{2}\right)$, Grupo $2\left(27.71 \pm 4.16 \mathrm{~kg} / \mathrm{m}^{2}\right) \mathrm{e}$ Grupo $3\left(26.54 \pm 4.66 \mathrm{~kg} / \mathrm{m}^{2}\right)$ para o IMC $(p<$ $0.05)$, conforme Tabela 1. Quando analisada a imagem corporal de todos os grupos, observouse diferença significativa entre a imagem corporal actual e desejada ( $p=0.001 ; p=0.001$; $p=0.001 ; p=0.001)$, correspondente à insatisfação com a imagem corporal. No entanto, não foi observada diferença significativa entre as mulheres adultas $v s$. meiaidade e idosas, praticantes e não praticantes quando comparado à imagem corporal actual $(p>0.05)$, conforme Tabela 2. 
56 | SVD Souto, JS Novaes, MD Monteiro, GR Neto, MI Mourão Carvalhal, E Coelho

Tabela 1

Comparação das variáveis antropométricas das mulheres adultas vs. meia-idade e idosas praticantes e não praticantes de hidroginástica

\begin{tabular}{|c|c|c|c|c|c|}
\hline \multirow{2}{*}{\multicolumn{6}{|c|}{ Grupo1 }} \\
\hline & & & & & \\
\hline Idade (anos) & & 20 & 49 & $36.89 \pm 8.12$ & 0.010 \\
\hline MC (kg) & & 45.70 & 104.70 & $67.65 \pm 10.32^{*}$ & 0.020 \\
\hline Estatura $(\mathrm{cm})$ & & 1.37 & 1.78 & $1.60 \pm 0.07$ & \\
\hline $\operatorname{IMC}\left(\mathrm{kg} / \mathrm{m}^{2}\right)$ & & 16.51 & 40.90 & $26.66 \pm 4.58$ & 0.010 \\
\hline \multicolumn{6}{|l|}{ Grupo 2} \\
\hline Idade (anos) & & 50 & 82 & $62.48 \pm 8.22$ & \\
\hline $\mathrm{MC}(\mathrm{Kg})$ & & 46.50 & 106.50 & $68.15 \pm 11.23^{*}$ & 0.001 \\
\hline Estatura $(\mathrm{cm})$ & & 1.43 & 1.74 & $1.57 \pm 0.07$ & \\
\hline $\operatorname{IMC}\left(\mathrm{kg} / \mathrm{m}^{2}\right)$ & & 19.38 & 42.66 & $27.71 \pm 4.16$ & \\
\hline \multicolumn{6}{|l|}{ Grupo 3} \\
\hline idade (anos) & & 50 & 83 & $61.28 \pm 7.99$ & \\
\hline $\mathrm{MC}(\mathrm{kg})$ & & 44.00 & 86.00 & $63.10 \pm 10.13$ & \\
\hline Estatura $(\mathrm{cm})$ & & 1.43 & 1.69 & $1.54 \pm 0.06$ & \\
\hline $\operatorname{IMC}\left(\mathrm{kg} / \mathrm{m}^{2}\right)$ & & 18.97 & 42.06 & $26.54 \pm 4.66$ & \\
\hline \multicolumn{6}{|l|}{ Grupo 4} \\
\hline Idade (anos) & & 20 & 51 & $35.19 \pm 9.14$ & \\
\hline MC (kg) & & 43.00 & 98.00 & $63.86 \pm 11.57$ & \\
\hline Estatura $(\mathrm{cm})$ & & 1.48 & 1.80 & $1.61 \pm 0.06$ & \\
\hline $\operatorname{IMC}\left(\mathrm{kg} / \mathrm{m}^{2}\right)$ & & 16.65 & 36.94 & $24.50 \pm 4.26 \dagger$ & \\
\hline \multicolumn{6}{|c|}{$\begin{array}{l}\text { Nota: * = diferença significativa para massa corporal de mulheres não praticantes e praticantes de hidroginástica; } \dagger=\text { diferença } \\
\text { significativa para o IMC entre o Grupo } 4 \text { e dos demais grupos; MC = massa corporal; IMC = índice de massa corporal. }\end{array}$} \\
\hline \multirow{2}{*}{\multicolumn{6}{|c|}{$\begin{array}{l}\text { Tabela } 2 \\
\text { Resultados para a percepção da imagem corporal das mulheres adultas vs. meia-idade e idosas praticantes e não } \\
\text { praticante de hidroginástica }\end{array}$}} \\
\hline & & & & & \\
\hline & & (\%) & $M \pm D P$ & Media Rank & Sig. \\
\hline \multicolumn{6}{|l|}{ Grupo 1} \\
\hline SA & $2(2.7 \%)$ & $73(97.3 \%)$ & $4.89 \pm 1.16^{*}$ & 36.62 & 0.001 \\
\hline SI & $14(18.7 \%)$ & $61(81.4 \%)$ & $3.15 \pm 0.75$ & & \\
\hline \multicolumn{6}{|l|}{ Grupo 2} \\
\hline SA & $1(1.3 \%)$ & $74(98.7 \%)$ & $4.61 \pm 1.33^{*}$ & 33.63 & 0.001 \\
\hline SI & $2(2.7 \%)$ & $73(97.4 \%)$ & $3.20 \pm 0.85$ & & \\
\hline \multicolumn{6}{|l|}{ Grupo 3} \\
\hline SA & $3(4.0 \%)$ & $72(96.0 \%)$ & $4.75 \pm 1.08^{*}$ & 30.40 & 0.001 \\
\hline SI & $17(22.6 \%)$ & $58(77.4 \%)$ & $3.20 \pm 0.82$ & & \\
\hline \multicolumn{6}{|l|}{ Grupo 4} \\
\hline SA & $3(4.0 \%)$ & $72(96.0 \%)$ & $4.43 \pm 1.19^{*}$ & 28.25 & 0.001 \\
\hline SI & $12(16.0 \%)$ & $63(84.0 \%)$ & $3.24 \pm 0.77$ & & \\
\hline
\end{tabular}

Nota: * diferença significativa entre a SA vs. SI; SA = silhueta atual; SI = silhueta ideal

$\mathrm{Na}$ análise das diferenças entre a imagem atual e a imagem desejada, ou seja, da insatisfação com a imagem corporal (em concordância com a apresentação do instrumento) observou-se valores médios para os respectivos grupos: Grupo 1( Nprat $\left._{20-40}\right)=1.7$; Grupo $2\left(\right.$ Nprat $\left._{50-82}\right)=1.60$; Grupo 3(Prat $\left.{ }_{50-83}\right)=$ 1.41 e Grupo 4(Prat $20-49)=1.19$. O que indica uma insatisfação com a imagem corporal em todos os grupos. Isto foi verificado pelo teste de associação, onde se observa um nível de -0.22 entre as SA e as SI, que aponta existir diferenças entre as percepções.

\section{DISCUSSÃo}

O presente estudo comparou a IC de mulheres adultas $v s$. meia-idade e idosas praticantes e não praticantes de hidroginástica. Os nossos resultados demonstram que a idade e a prática da hidroginástica não são factores determinantes da percepção da IC, além disso, o IMC apresentou diferenças significativas entre os grupos.

Nesta direção, Fermino, Pezzini, e Reis (2010) explicam que a insatisfação da IC não esta ligada apenas a composição corporal das mulheres, mas também com a massa corporal total. Isto pode explicar o motivo das não 
praticantes de hidroginástica se perceberem mais insatisfeitas uma vez que apresentam valores superiores. Similar ao nosso estudo, Kura, Ribeiro, Niquetti, e Tourinho Filho (2006) verificaram o índice de massa corporal entre 56 idosas praticantes de ginástica $(25.72 \pm 3.03$ $\left.\mathrm{kg} / \mathrm{m}^{2}\right)$ e hidroginástica $\left(27.81 \pm 4.47 \mathrm{~kg} / \mathrm{m}^{2}\right)$ e concluíram que os resultados estavam acima dos padrões normais, porém estavam dentro do padrão apresentado pela literatura (Garber et al., 2009), assim como os nossos resultados. Melo e Giavoni (2004) avaliaram e compararam os efeitos da ginástica aeróbia e da hidroginástica na composição corporal de mulheres idosas. Estes autores concluíram que a ginástica aeróbia gerou resultados mais positivos na composição corporal de mulheres idosas do que a hidroginástica, sendo que as praticantes de hidroginástica reduziram significativamente a proporção de gordura nas pernas. Analisando estes resultados e os do presente estudo, parece que a prática da hidroginástica auxilia na manutenção da composição corporal de mulheres idosas e pode influenciar na percepção da IC.

Os resultados desta investigação mostram que não existe diferença significativa para a SI e SA entre os subgrupos, porém, existem diferenças nos grupos (insatisfação com IC). Diversos estudos apresentam as evidências que as mulheres desejam uma massa corporal menos volumosa dentro de padrões da SI (Araújo \& Araújo, 2003; Loland, 2000; McLaren \& Gauvin, 2002).

Damasceno, Lima, Vianna, Vianna, e Novaes (2005) reportaram que $76 \%$ das mulheres entrevistadas estavam insatisfeitas com a IC. Esses resultados corroboram com os do presente estudo que apresentaram médias similares, mesmo sendo em intervenções diferentes (caminhada vs. hidroginástica), isto mostra que as mulheres independentes da faixa etária e das diferentes práticas de exercícios físicos estavam insatisfeitas com a IC, pois escolheram uma silhueta que representa um IMC que não condiz com sua SA.

Em relação à faixa etária, esses resultados mostram que as mulheres adultas apontam uma discordância para a SA, mas sem apresentarem diferenças significativas para SI, indicando que a idade não é um fator determinante na percepção que a mulher tem do seu corpo com a prática ou não de exercícios físicos. Essa constatação indica que mesmo nos subgrupos praticantes de hidroginástica pode ser observada insatisfação com a IC. Este achado reforça os resultados de estudos anteriores que expressam que a satisfação com a IC pose ser motivada pela prática do exercício físico, independentemente da idade (Fermino et al., 2010; Tiggemann, 2004). Parece que indivíduos motivados para a prática regular de exercícios físicos possuem uma satisfação superior com a IC quando comparados aos indivíduos não praticantes. Além disso, parece que a imagem ideal possa ser influenciada pelo padrão cultural, não variando com a faixa etária nem com a prática de exercícios. Analisando este contexto, parece que outros fatores externos podem influenciar na percepção da IC e que a influência positiva da prática sistemática do exercício físico pode ser inibida.

Analisando a IC, os achados do presente estudos demonstram que os grupos apresentam diferença significativa entre a imagem corporal actual e desejada. Porém, não apresenta diferença significativa entre os grupos. Estes resultados são corroborados pelo único estudo que avaliou a IC em praticantes de hidroginástica (Mazo et al., 2006). Estes autores avaliaram a autoestima e a autoimagem e a sua relação com os fatores motivacionais de ingresso e da permanência dos idosos em um programa de hidroginástica. Os autores concluíram que o os idosos apresentaram elevada autoestima e autoimagem e que houve diferença estatisticamente significativa entre os motivos de permanência no programa e na autoimagem corporal. Estes autores apontam que o programa de hidroginástica pode ser uma estratégia para melhoria da autoimagem e autoestima dos idosos e que isto possibilitava a permanência dos idosos no programa. Porém, fatores limitantes são encontrados no estudo de Mazo, Cardoso, e Aguiar (2006). Os autores não compararam os resultados com outro grupo e utilizaram um questionário sem validação, além disso, o estudo apresentou uma discrepância no número e no gênero de participante entre os sexos (53 mulheres e sete homens) o que poderia ter influenciado os resultados. 
O grupo das mulheres de meia-idade e idosas praticantes e não praticantes de hidroginástica manifestaram insatisfação com a IC o que se contrapõem aos relatos de Braggion, Matsudo, Matsudo, Andrade, e Araújo (2000). Estes autores afirmam que os indivíduos mais velhos são menos exigentes em relação a sua aparência. Nesse caso pode ser observado que as mulheres idosas desejam um estereotipo inferior ao real, mas mesmo assim, não se observa diferença estatística entre os grupos de praticantes e não praticantes de hidroginástica. Os resultados de Tribess, Junior, e Petroski (2010) apontam que $46 \%$ das mulheres adultas estavam satisfeitas com a SI, no entanto, no mesmo estudo os autores salientam que $54 \%$ estão insatisfeitas, o que converge para os resultados obtidos no presente estudo.

Finalmente, uma possível limitação do estudo refere-se ao seu delineamento de corte transversal, o que dificulta verificar a relação causa-efeito entre as variáveis. Outra limitação pode estar no método aplicado para estabelecer o nível de insatisfação, já que os desenhos não apresentam uma dimensão real e os indivíduos tendem em alguns casos superestimar as silhuetas reais (Damasceno, Lima, Vianna, Vianna, \& Novaes, 2005).

\section{CONCLUSÕES}

Os nossos resultados demonstram que a idade e a prática da hidroginástica não são factores determinantes da percepção que as mulheres têm da imagem corporal. Sugere-se que novos estudos sejam desenvolvidos analisando a percepção da imagem corporal em homens com diferentes faixas etárias e diferentes modalidades esportivas.

\section{Agradecimentos:}

Nada a declarar.

Conflito de Interesses:

Nada a declarar.

Financiamento:

Nada a declarar

\section{REFERÊNCIAS}

ACSM. (2009). American College of Sports Medicine Position Stand. Appropriate physical activity intervention strategies for weight loss and prevention of weight regain for adults. Medicine and Science in Sports and Exercise, 41(2), 459471. doi: 10.1249/MSS.0b013e3181949333

Araújo, D. S. M. S., \& Araújo, C. G. S. (2003). Selfperception and dissatisfaction with weight does not depend on the frequency of physical activity. Arquivos Brasileiros de Cardiologia, 80(3), 243249.

Assis, R. S., Junior, L. F. S. S., Santos, L. R., \& Navarro, A. C. (2007). The hidroginástica improves the physical conditioning of the aged ones. Revista Brasileira de Prescrição e Fisiologia do Exercício, 1(5), 62-75.

Baum, G. (2000). Aquaeróbica - Manual de treinamento (1 ed.): Manole.

Braggion, G. F., Matsudo, S. M. M., Matsudo, V. K. R., Andrade, E. L., \& Araújo, T. L. (2000). Comparação das variáveis antropométricas e acordo com o grau de satisfação com a aparência corporal em senhoras ativa acima de 50 anos. Revista Brasileira de Ciencia e Movimento, 8.

Cash, T. F., \& Pruzinsky, T. E. (1990). Body images: Development, deviance, and change. New York: Guilford Press.

Damasceno, V. O., Lima, J. R. P., Vianna, J. M., Vianna, V. R. Á., \& Novaes, J. S. (2005). Ideal physical type and body image satisfaction of regular walkers. Revista Brasileira de Medicina do Esporte, 11(3), 181-186.

Etchepare, L., Pereira, É. F., Graup, S., \& Zinn, J. L. (2003). Terceira idade: aptidão física de praticantes de hidroginástica. Efdeportes Revista Digital, 61(9).

Fermino, R. C., Pezzini, M. R., \& Reis, R. S. (2010). Motivos para prática de atividade física e imagem corporal em frequentadores de academia. Revista Brasileira de Medicina do Esporte, 16(1), 18-23.

Garber, C. E., Blissmer, B., Deschenes, M. R., Franklin, B. A., Lamonte, M. J., Lee, I.-M., ... Swain, D. P. (2011). Quantity and Quality of Exercise for Developing and Maintaining Cardiorespiratory, Musculoskeletal, and Neuromotor Fitness in Apparently Healthy Adults: Guidance for Prescribing Exercise. Medicine \& Science in Sports \& Exercise, 43(7), 1334-1359. https://doi.org/10.1249/MSS.0b013e318213fefb

Hausenblas, H. A., \& Fallon, E. A. (2006). Exercise and body image: A meta-analysis. Psychology and Health, 21(1), 33-47.

Kura, G. G., Ribeiro, L. S. P., Niquetti, R., \& Tourinho Filho, H. (2006). Nível de atividade física, IMC e índices de força muscular estática entre idosas praticantes de hidroginástica e ginástica. Revista Brasileira de Ciências do Envelhecimento Humano, 1(2), 30-40.

Loland, N. W. (2000). The aging body: Attitudes toward bodily appearance among physically active and inactive women and men of different ages. 
Journal of Aging and Physical Activity 8(3), 197213.

Matsuo, R. F., Velardi, M., Brandão, M. R. F., \& Jesus, M. M. L. (2007). Older women's body image and physical activity. Revista Mackenzie de Educação Física e Esporte, 6(1), 37-43.

Mazo, G. Z., Cardoso, F. L., \& Aguiar, D. L. (2006). Water gymnastics program for elderly: motivation, self-steam and self-image. Revista Brasileira de Cineantropometria e Desempenho Humano, 8(2), 67-72.

McLaren, L., \& Gauvin, L. (2002). Neighbourhood level versus individual level correlates of women's body dissatisfaction: toward a multilevel understanding of the role of affluence. Journal of Epidemiology and Community Health, 56(3), 193-199.

Melo, G. F., \& Giavoni, A. (2004). Comparação dos efeitos da ginástica aeróbica e da hidroginástica na composição corporal de mulheres idosas. Revista Brasileira de Ciência e Movimento, 12(2), 13-18.

Ricciardelli, L. A., McCabe, M. P., \& Banfield, S. (2000). Body image and body change methods in adolescent boys: Role of parents, friends and the media. Journal of Psychosomatic Research, 49(3), 189-197.

Sato, D., Kaneda, K., Wakabayashi, H., \& Nomura, T. (2007). The water exercise improves healthrelated quality of life of frail elderly people at day service facility. Quality of Life Research 16(10), 1577-1585.

Sato, D., Seko, C., Hashitomi, T., Sengoku, Y., \& Nomura, T. (2015). Differential effects of waterbased exercise on the cognitive function in independent elderly adults. Aging Clinical and Experimental Research, 27(2), 149-159. https://doi.org/10.1007/s40520-014-0252-9
Scatolin, H. G. (2012). A imagem do corpo: as energias construtivas da psique. Psicologia Revista. Revista da Faculdade de Ciências Humanas e da Saúde, 21(1), 115-120.

Schuch, F. B., Pinto, S. S., Bagatini, N. C., Zaffari, P., Alberton, C. L., Cadore, E. L., . . Kruel, L. F. M. (2014). Water-Based Exercise and Quality of Life in Women: The Role of Depressive Symptoms. Women Health, 54(2), 161-175. doi: $10.1080 / 03630242.2013 .870634$

Stunkard, A. J., Sørensen, T., \& Schulsinger, F. (1983). Use of the Danish Adoption Register for the study of obesity and thinness. Em S. S. Kety, L. P. Rowland, R. L. Sidman, \& S. W. Matthysse (Eds.), The Genetics of Neurological and Psychiatric Disorders (Vol. 60, pp. 115-120). New York: Raven Press.

Tiggemann, M. (2004). Body image across the adult life span: Stability and change. Body Image, 1(1), 29-41.

Tribess, S., Junior, J. S. V., \& Petroski, E. L. (2010). Estado nutricional e percepção da imagem corporal de mulheres idosas residentes no nordeste do Brasil. Ciência \& Saúde Coletiva, 15(1), 31-38.

Veggi, A. B., Lopes, C. S., Faerstein, E., \& Sichieri, R. (2004). Índice de massa corporal, percepção do peso corporal e transtornos mentais comuns entre funcionários de uma universidade no Rio de Janeiro. Revista Brasileira de Psiquiatria, 26(4), 242-247.

Volkwein, K., \& McConatha, J. T. (1997). Cultural contours of the body - The impact of age and fitness. Em R. Lidor \& E. Bar-Eli (Eds.), Innovations in sport psychology: Linking theory and practice (pp. 744-746). Tel-Aviv: ISSP. 